\title{
Tác động của năng lực lãnh đạo đến kết quả hoạt động doanh nghiệp theo quan điểm thẻ điểm cân bằng tại các doanh nghiệp vừa và nhỏ ở Thành phố Hồ Chí Minh
}

\section{Impact of leadership capacities on enterprise performance results by viewpoints of balanced points in small and medium enterprises in $\mathrm{Ho}$ Chi Minh City}

\author{
Bùi Thị Minh Thu ${ }^{1 *}$, Trần Thị Ngân Hà ${ }^{1}$ \\ ${ }^{1}$ Trường Đại học Nội vụ Hà Nội Phân hiệu Quảng Nam, Việt Nam \\ *Tác giả liên hệ, Email: thubtmgv@gmail.com
}

\begin{tabular}{l} 
THÔNG TIN \\
\hline DOI:10.46223/HCMCOUJS. \\
econ.vi.14.2.488.2019 \\
Ngày nhận: 04/03/2019 \\
Ngày nhận lại: 03/05/2019 \\
Duyệt đăng: 20/06/2019
\end{tabular}

Tù khóa:

doanh nghiệp vừa và nhỏ, kết quả hoạt động doanh nghiệp, năng lực lãnh đạo

Keywords:

business performance results, leadership capacity, small and medium enterprises

\section{TÓM TẮT}

Số liệu từ Tổng cục Thống kê cho thấy năm 2015 có 39.056 doanh nghiệp vừa và nhỏ gặp khó khăn buộc phải tạm ngừng hoạt động, tăng $2 \%$ so với năm 2014, đặc biệt là ở thị trường rộng lớn Thành phố Hồ Chí Minh. Nhận thức được tầm quan trọng này, nghiên cứu đã thu thập được 280 quan sát ở các doanh nghiệp vừa và nhỏ và kiểm định thông qua mô hình SEM mối quan hệ tác động của năng lực lãnh đạo đến kết quả hoạt động doanh nghiệp theo quan điểm thẻ điểm cân bằng tại các doanh nghiệp vừa và nhỏ ở Thành phố Hồ Chí Minh. Kết luận của nghiên cứu có thể rút ra là "Khi giám đốc doanh nghiệp có mức độ đáp ứng về các năng lực lãnh đạo càng tốt thì kết quả hoạt động của doanh nghiệp cũng sẽ khả quan hơn".

\section{ABSTRACT}

Data from the General Statistics Office of Vietnam show that there were 39,056 small and medium enterprises facing difficulties to temporarily suspend operations in 2015, an increase of 2\% compared to 2014, especially in the large market of Ho Chi Minh City. Recognizing this importance, the study has collected 280 observations in small and medium enterprises and accredited through SEM model about the impact of leadership on business performance according to Viewpoints of Balanced Points at small and medium enterprises in Ho Chi Minh City. The conclusion of the study can be drawn as "The more competent the enterprise director's responsiveness levels to the leadership capacities are, the better the performance of the business is". 


\section{1. Đặt vấn đề}

Thực tiễn đã chỉ ra rằng lãnh đạo mà cụ thể là năng lực lãnh đạo của người đứng đầu là nhân tố quyết định thành công của doanh nghiệp. Theo học giả Bennis (2009), lãnh đạo là quá trình gây ảnh hưởng mang tính xã hội nhằm tìm kiếm sự tham gia tự nguyện của cấp dưới trong việc thực thi mục tiêu, sứ mệnh. Năng lực lãnh đạo chính là tổng hợp các kiến thức, kỹ năng, kinh nghiệm và hành vi, thái độ nhằm biến tổ chức, doanh nghiệp thành một khối kết dính, thống nhất, đảm bảo cạnh tranh thành công trên thương trường. Hiện nay, doanh nghiệp nhỏ và vừa chiếm tới $96 \%$ số doanh nghiệp (DN) ở Việt Nam nhưng quy mô, năng lực cạnh tranh vẫn còn yếu. Kết quả tổng điều tra kinh tế năm 2017 cho thấy, toàn Thành phố Hồ Chí Minh, trong tổng số $171.655 \mathrm{DN}$ đang hoạt động, chỉ có 64.607 DN hoạt động có lãi, chiếm 37,81\%; 96.936 DN bị thua lỗ, chiếm $56,49 \%$; số còn lại kinh doanh hòa vốn. Tỷ lệ các DN thua lỗ sau 5 năm có xu hướng tăng lên, đặc biệt là $\mathrm{DN}$ ngoài nhà nước đã tăng $2,87 \%$, số $\mathrm{DN}$ có lãi lại giảm từ 43,30\% năm 2011 xuống còn 37,41\% năm 2016. Tương tự, với $\mathrm{DN}$ nhà nước, tỷ lệ $\mathrm{DN}$ sản xuất kinh doanh có lãi là $81,79 \%$, giảm $0,71 \%$, tỷ lệ $\mathrm{DN}$ bị thua lỗ từ $16,6 \%$ năm 2011 tăng lên 17,65\% năm 2016. Một trong những nguyên nhân dẫn đến tình trạng trên là do công tác quản trị nhân sự còn yếu kém, đặc biệt là việc phát triển năng lực quản trị, năng lực lãnh đạo của đội ngũ giám đốc nói riêng và đội ngũ nhà quản trị trong doanh nghiệp nói chung chưa được chú trọng cũng là nguyên nhân khiến cho các doanh nghiệp nhỏ và vừa khó tiếp tục phát triển. Do đó nghiên cứu "Tác động của năng lục lãnh đạo đến kết quả hoạt động doanh nghiệp theo quan điểm thẻ điểm cân bằng tại các doanh nghiệp vù̀a và nhỏ ở khu vục thành phố Hồ Chí Minh" có ý nghĩa thiết thực nhằm hệ thống hóa các vấn đề lý luận, tìm hiểu thực trạng, phát hiện những tác động trong năng lực lãnh đạo đến kết quả hoạt động của doanh nghiệp, từ đó đề xuất giải pháp nhằm nâng cao năng lực lãnh đạo của đội ngũ này trong thời gian tới.

\section{Cơ sở lí thuyết}

\subsection{Khái niệm về lãnh đạo}

Khái niệm về lãnh đạo có thể được tiếp cận dưới góc độ tố chất, góc độ hành vi, cũng có thể được tiếp cận dưới góc độ gây ảnh hưởng hay góc độ sự tương tác qua lại. Theo nhà nghiên cứu Bennis (2009), lãnh đạo là một quá trình trong đó một đối tượng thuyết phục cấp dưới hành động theo như mong muốn. Schein (1992), lãnh đạo là khả năng bứt phá khỏi văn hóa nhằm thực hiện một quá trình thay đổi tiến hóa mang tính thích ứng cao. Kabeer, Othman, và Dsilva (2012), lãnh đạo là quá trình gây ảnh hưởng đến người khác để họ hiểu và đồng ý về những công việc cần thực hiện và thực hiện nó như thế nào một cách hiệu quả, là quá trình tạo điều kiện thuận lợi cho các cá nhân và tập thể nỗ lực đạt được mục tiêu của tổ chức đề ra. Bennis (2009), lãnh đạo là quá trình gây ảnh hưởng mang tính xã hội nhằm tìm kiếm sự tham gia tự nguyện của cấp dưới để thực hiện một cách tốt nhất các mục tiêu của tổ chức. Theo nghiên cứu dù nhìn nhận theo cách nào, thì một nhà lãnh đạo phải đảm bảo được 3 yếu tố: khả năng tạo tầm nhìn, khả năng truyền cảm hứng và khả năng gây ảnh hưởng. Như vậy, theo cách hiểu đơn giản nhất, lãnh đạo là quá trình ảnh hưởng, tác động (bằng cách tạo điều kiện, môi trường, truyền cảm hứng) đến con người để tìm kiếm sự tham gia tự nguyện của họ nhằm đạt mục tiêu, nhiệm vụ, sứ mạng của nhóm, của tổ chức.

\subsection{Khái niệm năng lục lânh đạo của giám đốc $D N N V V$}

Theo Doh (2003), có nhiều quan niệm khác nhau về năng lực lãnh đạo: Năng lực lãnh đạo là khả năng tạo ra động lực và hứng khởi cho bản thân và sau nữa là truyền sự hứng khởi cho người khác. Năng lực lãnh đạo là khả năng giành được sự ủng hộ và nỗ lực tối đa từ các 
thành viên trong tổ chức. Năng lực lãnh đạo là tổng hợp các tố chất, kiến thức, hành vi thái độ, kỹ năng mà nhà lãnh đạo cần có để hoàn thành nhiệm vụ của mình. Năng lực lãnh đạo là khả năng của cá nhân nhằm gây ảnh hưởng, thúc đẩy và khiến người khác cống hiến vì hiệu quả và thành công của tổ chức. Theo nghiên cứu: "Năng lực lãnh đạo của giám đốc các DNNVV trong nghiên cứu được hiểu đó là sự tổng hợp các kiến thức, kỹ năng và phẩm chất, thái độ mà một giám đốc doanh nghiệp cần có trong hoạt động lãnh đạo bản thân, lãnh đạo đội ngũ cấp dưới, lãnh đạo tổ chức nhằm đạt được mục tiêu của doanh nghiệp đã định ra".

\subsection{Kết quả hoạt động doanh nghiệp theo quan điểm thẻ điểm cân bằng}

Thẻ điểm cân bằng (Balanced Scorecard) lần đầu tiên được giới thiệu vào năm 1992 bởi hai giáo sư đại học Harvard là Kaplan và Norton với mục đích là thúc đẩy và đo lường hiệu quả hoạt động của các đơn vị kinh doanh. BSC nhanh chóng được hàng ngàn các doanh nghiệp, các cơ quan chính phủ, các tổ chức phi lợi nhuận khắp nơi trên thế giới áp dụng trong đó có Việt Nam. Gần 20 năm sau, trong kết quả khảo sát toàn cầu về các công cụ quản lý năm 2011 do hãng tư vấn Bain công bố, Thẻ điểm cân bằng đã lọt vào tốp 10 công cụ quản lý được sử dụng rộng rãi nhất trên thế giới (vị trí thứ 6 ). Thẻ điểm cân bằng $\mathrm{BSC}$ bao gồm 4 thành phần:

- Phương diện Tài chính: Một số các chỉ số đo lường phương diện tài chính thường được sử dụng là: Chỉ tiêu tỷ lệ hoàn vốn đầu tư (ROI), Tỷ suất sinh lợi trên tổng tài sản (ROA), Tỷ suất sinh lợi trên doanh thu (ROS), Tỷ suất sinh lời trên vốn chủ sở hữu (ROE), Lợi nhuận còn lại (thặng dư) RI, Giá trị kinh tế tăng thêm (EVA - Economic Value Added);

- Phương diện Khách hàng: Một số các chỉ số đo lường phương diện khách hàng thường được sử dụng: sự hài lòng của khách hàng; lòng trung thành của khách hàng; thị phần; tỷ lệ khách hàng tăng thêm; doanh thu trên từng kênh...

- Phương diện Quy trình nội bộ: Một số các chỉ số đo lường phương diện quy trình nội bộ thường được sử dụng: Các chỉ tiêu chi phí cho nghiên cứu, thời gian giải quyêt đơn hàng, công suất máy móc thiết bị, thời gian bảo trì, phục vụ hay khắc phục sản phẩm lỗi...

- Phương diện Đào tạo - Phát triển: Một số các chỉ số đo lường phương diện đào tạo và phát triển thường được sử dụng: số nhân viên đã qua huấn luyện, đào tạo; tỷ lệ thay thế nhân viên; sự hài lòng của nhân viên; tỷ lệ \% nhân viên có bằng cấp cao...

Trong nghiên cứu, tác giả đã lựa chọn sẽ đánh giá sự ảnh hưởng của năng lực lãnh đạo của giám đốc DNNVV thông qua các chỉ số kết quả hoạt động của doanh nghiệp theo Thẻ điểm cân bằng $\mathrm{BSC}$.

\subsection{Tình hình kinh tế Thành phố Hồ Chí Minh}

Tổng điều tra kinh tế năm 2017 cho thấy, toàn Thành phố Hồ Chí Minh hiện có 633.637 đơn vị kinh tế, hành chính, sự nghiệp và cơ sở tôn giáo, tín ngưỡng (chưa bao gồm 13.076 cơ sở, chi nhánh và văn phòng trực thuộc doanh nghiệp), tăng 26,99\% so với năm 2011, tương đương tăng 134.421 đơn vị và bình quân hàng năm tăng khoảng 4,90\%. Tổng số lao động trong các đơn vị gần 4,1 triệu người, tăng $19,04 \%$ so với năm 2011, tốc độ tăng bình quân hàng năm là 3,55\%. Tốc độ phát triển các cơ sở kinh tế tăng cao hơn so với các đơn vị hành chính sự nghiệp, với mức tăng là $26,99 \%$ về số cơ sở và $10,04 \%$ về số lao động so với năm 2011 . Cụ thể, đối với khối doanh nghiệp (DN), hợp tác xã (HTX), tính đến ngày 31-12-2016, toàn TP có 172.979 DN, HTX (gọi chung là DN), tăng 61,86\%, tương ứng 66.089 DN so với năm 2011. Trong đó, loại hình $\mathrm{DN}$ ngoài nhà nước luôn có bước phát triển vượt bậc so với các loại hình còn lại, đạt $65,35 \%$, chiếm $97,36 \%$ trong tổng số $\mathrm{DN}$ toàn $\mathrm{TP}$. Tổng nguồn vốn của khối $\mathrm{DN}$ 
này tăng 2,11 lần, tương ứng 3.262.149 tỷ đồng, trong đó vốn chủ sở hữu tăng 3,23 lần, chỉ tiêu doanh thu thuần gấp 1,56 lần so năm 2011. Đây cũng là khu vực tạo ra nhiều việc làm mới cho người lao động. Số liệu điều tra cũng chỉ ra, mặc dù số lượng DN phát triển nhanh nhưng quy mô $\mathrm{DN}$ thì chủ yếu là $\mathrm{DN}$ nhỏ và siêu nhỏ. Xét về hiệu quả thông qua chỉ tiêu lợi nhuận còn khá hạn chế.

\section{Phương pháp nghiên cứu}

\subsection{Giới thiệu nghiên cúu}

Để xây dựng câu hỏi khảo sát thì nhóm các chuyên gia bao gồm có 5 thầy cô với trình dộ nghiên cứu sâu về quản trị nhân lực ở Đại học Kinh tế Đà Nẵng, Đại học kinh tế Huế, 3 giám đốc doanh nghiệp vừa và nhỏ đã cung cấp các thông tin, dữ liệu để xác định các yếu tố hình bảng câu hỏi khảo sát về năng lực lành đạo tác động đến kết quả hoạt động doanh nghiệp theo quan điểm thẻ điểm cân bằng. Trong phần nghiên cứu định tính này, một số tên gọi đã được các chuyên gia góp ý để chỉnh sửa cho phù hợp với đặc điểm và điều kiện của doanh nghiệp vừa và nhỏ tại Thành phố Hồ Chí Minh.

Để phân tích tác động của năng lực lãnh đạo đến kết quả hoạt động doanh nghiệp theo quan điểm thẻ điểm cân bằng tại các doanh nghiệp vừa và nhỏ ở khu vực Thành phố Hồ Chí Minh, nghiên cứu sử dụng kỹ thuật phân tích được xây dựng dựa trên nền tảng lý thuyết mô hình phương trình cấu trúc SEM (Structural Equation Modeling) và sự hỗ trợ của phần mềm SPSS 22 và phần mềm AMOS (Analysis of Moment Structures). Với kỹ thuật phân tích này bỏ qua đa cộng tuyến và sự tin cậy của dữ liệu thị trường cũng được xem xét thông qua các sai số đo lường. Đánh giá độ tin cậy thang đo thông qua đại lượng Cronbach’s Alpha, phân tích nhân tố khám phá EFA (Exploratory Factor Analysis). Kỹ thuật phân tích nhân tố khẳng định CFA, Kỹ thuật phân tích mô hình cấu trúc SEM.

\subsection{Phưong pháp thu thập số liệu}

\subsubsection{Xác định cõ̃ mẫu}

Theo các nhà nghiên cứu Hair, Anderson, Tatham, và Black (1998), thì để chọn kích thước quan sát nghiên cứu phù hợp đối với phân tích nhân tố khám phá EFA cỡ quan sát tối thiểu $\mathrm{N}>5^{*} \mathrm{x}$ (x: là tổng số biến quan sát). Theo Tabachnick và Fidell (1996) để tiến hành phân tích hồi quy một cách tốt nhất thì cỡ quan sát tối thiểu cần đạt được tính theo công thức $\mathrm{N}>$ $50+8 \mathrm{~m}$ (trong đó $\mathrm{m}$ là biến độc lập). Phù hợp với nghiên cứu của mình thì tác giả sử dụng số quan sát ứng với 13 thang đo quan sát của kết quả hoạt động doanh nghiệp, 34 biến của năng lực lãnh đạo của doanh nghiệp là: $\mathrm{N}>\max (5 \times 51 ; 50+9 \times 13)=(255,167)=255$ quan sát. Do các DNVVN Thành phố Hồ Chí Minh có số lượng nhiều nên tác giả lấy nhiều số liệu quan sát để đảm bảo tính chính xác hơn cho dữ liệu nghiên cứu, cuối cùng tổng quan sát thu được là 280 quan sát là phù hợp với nghiên cứu.

\subsubsection{Phương pháp chọn mẫu}

Việc lựa chọn phương pháp chọn quan sát ở đây là phương pháp chọn quan sát theo hạn ngạch (quota) của các nhóm đối tượng được phân chia theo biến số địa bàn, khu vực. Đối tượng tham gia trong nghiên cứu này bao gồm người lao động của các doanh nghiệp vừa và nhỏ tại Thành phố Hồ Chí Minh với chức vụ lãnh đạo từ cấp trưởng phòng trở lên. Số lượng quan sát điều tra ở dựa trên số lượng doanh nghiệp vừa và nhỏ tại Thành phố Hồ Chí Minh là 171.655 
doanh nghiệp, tổng cộng 300 quan sát phát ra, thu vào là 280 quan sát. Việc phỏng vấn đáp viên theo hình thức phát trực tiếp tại nơi làm việc là 95 phiếu, gửi mail là 205 phiếu.

\subsection{Phưong pháp xử lý số liệu}

Kỹ thuật phân tích của nghiên cứu được xây dựng dựa trên nền tảng lý thuyết mô hình phương trình cấu trúc SEM (Structural Equation Modeling) và sự hỗ trợ của phần mềm SPSS 22 và phần mềm AMOS (Analysis of Moment Structures). Với kỹ thuật phân tích này sẽ bỏ qua đa cộng tuyến trong mô hình và sự tin cậy của dữ liệu thị trường cũng được xem xét thông qua các sai số đo lường. Đánh giá độ tin cậy thang đo thông qua đại lượng Cronbach’s Alpha, phân tích nhân tố khám phá EFA (Exploratory Factor Analysis). Kỹ thuật phân tích mô hình cấu trúc SEM đã được sử dụng rộng rãi trong các lĩnh vực nghiên cứu như tâm lý học, xã hội học và trong lĩnh vực quản lý. Như vậy trong nghiên cứu sẽ sử dụng phần mềm AMOS để kiểm tra SEM với các thông số phù hợp mô hình và hệ số tương quan $>0.5$.

\subsection{Mô hình nghiên cứu}

Các yếu tố cấu thành năng lực lãnh đạo của giám đốc DNNVV ảnh hưởng đến kết quả hoạt động của doanh nghiệp. Có khá nhiều quan điểm của các nhà nghiên cứu chỉ ra mối quan hệ giữa giám đốc doanh nghiệp và kết quả hoạt động của doanh nghiệp. Peterson và Sorenson (2005) có nhiều lý thuyết giải thích ảnh hưởng của giám đốc doanh nghiệp tới kết quả hoạt động của doanh nghiệp. Nghiên cứu của Bass (1990) cũng đề cập đến các kiến thức lãnh đạo như kiến thức hiểu biết chung, kiến thức liên quan đến nghề nghiệp... là những thành phần tất yếu khi nói đến năng lực lãnh đạo. Như vậy, kiến thức lãnh đạo là một yếu tố cấu thành năng lực lãnh đạo của giám đốc DNNVV nên tác giả đưa ra giả thuyết:

H1: Kiến thức lãnh đạo của giám đốc DNNVV có quan hệ thuận chiều với kết quả hoạt động của doanh nghiệp

Trong một nghiên cứu của Akhmad và cộng sự (2013) thì kỹ năng và năng lực của chủ doanh nghiệp là nguyên liệu không thể thiếu để tạo nên sự thành công của các doanh nghiệp cũng như quyết định hiệu quả kinh doanh của doanh nghiệp. Hayton (2015) cũng đã khảo sát trên 2.500 DNNVV ở Anh và kết quả cho thấy các kỹ năng của giám đốc DNNVV (bao gồm các kỹ năng lãnh đạo, kỹ năng kinh doanh, kỹ năng kỹ thuật, và các kỹ năng tổ chức) có ảnh hưởng đến kết quả hoạt động của doanh nghiệp (doanh thu, thị phần, năng suất...). Theo đó, tác giả đưa ra giả thuyết:

H2: Kỹ năng lãnh đạo của giám đốc DNNVV có quan hệ thuận chiều với kết quả hoạt động của doanh nghiệp

Do \& Nguyen (2015) đã nghiên cứu và chỉ ra rằng có mối quan hệ ảnh hưởng giữa tố chất lãnh đạo như sự đồng cảm, tính nhất quán, tính trung thực, sự mềm dẻo, niềm tin...đối với yếu tố lãnh đạo hiệu quả. Boal (2004) cho rằng có những tố chất nhất định của lãnh đạo ảnh hưởng tới kết quả hoạt động của doanh nghiệp. Giả thuyết thứ 3:

H3: Phẩm chất lãnh đạo của giám đốc DNNVV có quan hệ thuận chiều với kết quả hoạt động của doanh nghiệp

Dựa theo các giả thiết và tổng quan tài liệu, mô hình nghiên cứu được đề xuất: 


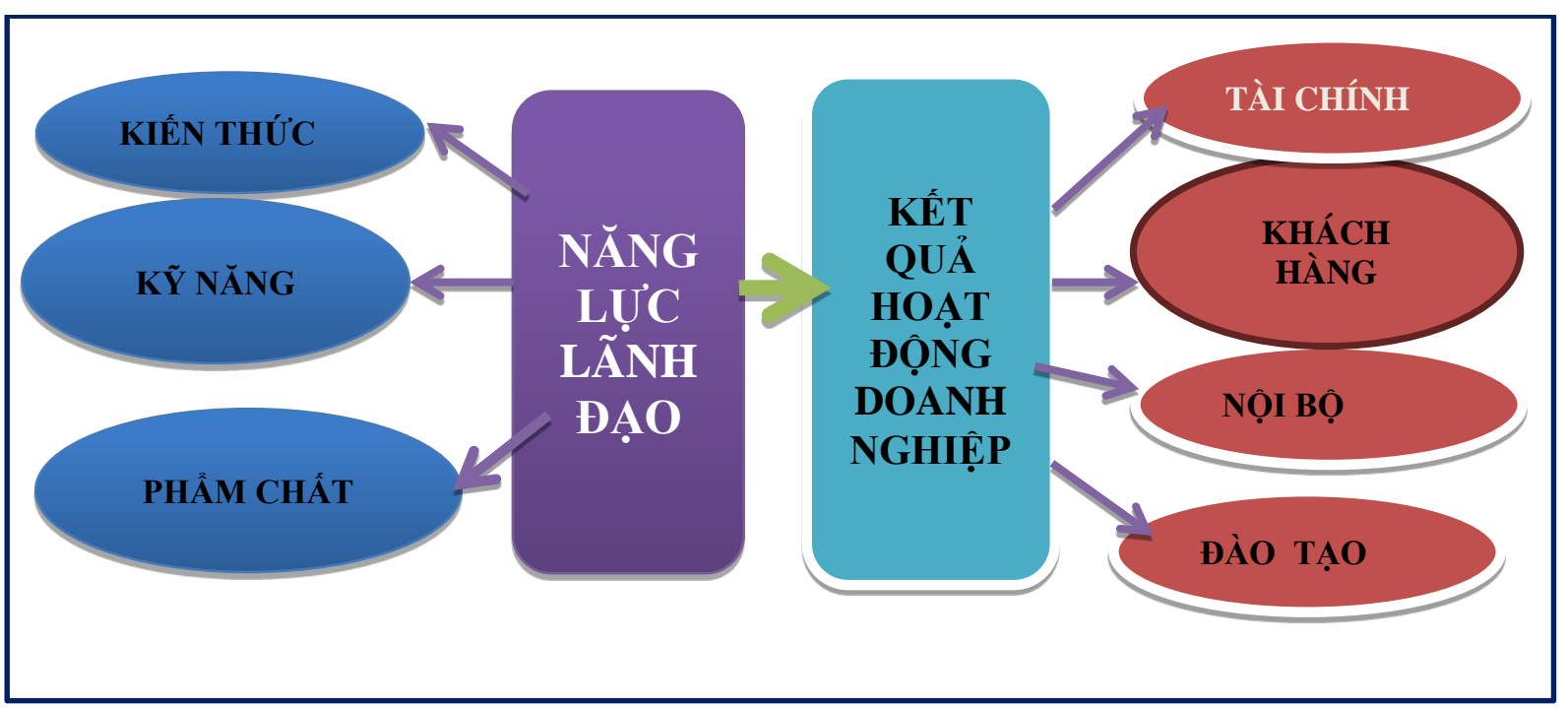

Nguồn: Đề xuất của tác giả

Hình 1. Mô hình nghiên cứu đề xuất

\subsection{Lụa chọn và phát triển thang đo}

\subsubsection{Thang đo kêt quả hoạt động của doanh nghiệp}

Tác giả kế thừa thang đo của Nguyen (2009). Theo đó, kết quả hoạt động của doanh nghiệp sẽ được đo lường thông qua thẻ điểm cân bằng $\mathrm{BSC}$ với bốn phương diện Tài chính Khách hàng - Qui trình nội bộ - Đào tạo và phát triển. Trong đó tác giả bổ sung thêm yếu tố "Hệ thống công nghệ thông tin hiện đại" và "Các chương trình hoạt động xã hội của doanh nghiệp tăng" vào phương diện Qui trình nội bộ; tiêu chí "Số lượng khách hàng mới tăng" vào phương diện Khách hàng, và tiêu chí "Hoạt động chăm sóc khách hàng hiệu quả" vào phương diện Qui trình nội bộ. Thang đo Likert được sử dụng để đo lường mức độ đồng ý của người điều tra với các nhận định về kết quả năm này so với mục tiêu mà doanh nghiệp đã đặt ra từ trước (từ mức 1đến mức 5 theo ý kiến chuyên gia).

\section{Bảng 1}

Thang đo kết quả hoạt động doanh nghiệp

\begin{tabular}{|c|c|c|c|}
\hline Thang do & & Thang do & \\
\hline $\begin{array}{c}\text { ĐÀO } \\
\text { TẠO (3) }\end{array}$ & $\begin{array}{l}\text { DTPT1.Số lượng người lao động } \\
\text { đã qua đào tạo tăng } \\
\text { DTPT2. Sự hài lòng của người } \\
\text { lao động về doanh nghiệp cao } \\
\text { DTPT3.Số lượng các vụ sai } \\
\text { phạm liên quan đến đạo đức nghề } \\
\text { nghiệp của người lao động giảm }\end{array}$ & $\begin{array}{c}\text { QUY } \\
\text { TRİNH } \\
\text { NộI Bộ } \\
\text { (5) }\end{array}$ & $\begin{array}{l}\text { QTNB1. Hệ thống công nghệ thông tin } \\
\text { hiện đại hơn; QTNB2. Công suất máy } \\
\text { móc thiết bị đạt chỉ tiêu; QTNB3. Hoạt } \\
\text { động chăm sóc khách hàng hiệu quả hơn } \\
\text { QTNB4. Số lượng sản phẩm mới, dịch } \\
\text { vụ mới tăng; QTNB5. Các chương trình } \\
\text { hoạt động xã hội doanh nghiệp tăng }\end{array}$ \\
\hline $\begin{array}{c}\text { KHÁCH } \\
\text { HÀNG } \\
\text { (3) }\end{array}$ & $\begin{array}{l}\text { KH1.Thị phần của DN tăng } \\
\text { KH2. lượng khách hàng mới tăng } \\
\text { KH3. Số lượng khách hàng hài } \\
\text { lòng về DN tăng }\end{array}$ & $\begin{array}{l}\text { TÀI } \\
\text { CHÍNH } \\
(2)\end{array}$ & $\begin{array}{l}\text { TC1 Doanh số tăng } \\
\text { TC2 Lợi nhuận tăng }\end{array}$ \\
\hline
\end{tabular}

Nguồn: Đề xuất của tác giả 


\subsubsection{Thang đo năng lục lãnh đạo doanh nghiệp}

\section{Bảng 2}

Thang đo năng lực lãnh đạo doanh nghiệp

\begin{tabular}{|c|c|}
\hline đo & \\
\hline $\begin{array}{l}\text { Kiến thức } \\
\text { lãnh đạo } \\
\text { (14) }\end{array}$ & $\begin{array}{l}\text { KT1 Kiến thức về lãnh vực ngành nghề kinh doanh; KT2 Kiến thức về văn hóa, xã } \\
\text { hội;KT3 Kiến thức chính trị, pháp luật; KT4 Kiến thức về lãnh đạo bản thân; KT5 Kiến } \\
\text { thức về chiến lược kinh doanh ; KT6 Kiến thức về quản trị nhân lực; KT7 Kiến thức } \\
\text { về marketing; KT8 Kiến thức về tài chính, kế toán; KT9 Kiến thức về quản trị sản xuất, } \\
\text { dịch vụ... KT10 Kiến thức về trách nhiệm xã hội của doanh nghiệp; KT11 Kiến thức } \\
\text { về văn hóa doanh nghiệp; KT12 Kiến thức về quản trị sự thay đồi, quản trị rủi ro; KT13 } \\
\text { Kiến thức về hội nhập quốc tế; KT14 Kiến thức ngoại ngữ, tin học }\end{array}$ \\
\hline $\begin{array}{l}\text { Kỹ năng } \\
\text { lãnh đạoo } \\
\quad(\mathbf{1 4})\end{array}$ & $\begin{array}{l}\text { KN1 Kỹ năng thấu hiểu bản thân; KN2 Kỹ năng cân bằng công việc và cuộc sống } \\
\text { KN3 Kỹ năng học hỏi; KN4 Kỹ năng giải quyết vấn đề; KN5 Kỹ năng giao tiếp lãnh } \\
\text { đạo; KN6 Kỹ năng động viên khuyến khích; KN7 Kỹ năng phát triển đội ngũ; KN8 Kỹ } \\
\text { năng gây ảnh hường và xây dựng hình ảnh; KN9 Kỹ năng thiết lập và lãnh đạo nhóm; } \\
\text { KN10 Kỹ năng xây dựng tầm nhìn và lập chiến lược; KN11 Kỹ năng tồ chức và triển } \\
\text { khai công việc ;KN12 Kỹ năng huy động và phối hợp các nguồn lực; KN13 Kỹ năng } \\
\text { khởi xướng sự thay đổi;KN14 Kỹ năng xây dựng và phát triển văn hóa doanh nghiệp }\end{array}$ \\
\hline $\begin{array}{l}\text { Phẩm chất } \\
\text { lãnh đạo } \\
\text { (10) }\end{array}$ & $\begin{array}{l}\text { PC1 Nhìn xa trông rộng; PC2 Tính mạo hiểm và quyết đoán; PC3 Ham học hỏi } \\
\text { PC4 Tư duy đổi mới và sáng tạo; PC5 Linh hoạt và nhạy bén; PC6 Trách nhiệm ;PC7 } \\
\text { Tính bao quá; PC8 Đạo đức nghề nghiệp; PC9 Tính kiên nhẫn ;PC10 Tự tin }\end{array}$ \\
\hline
\end{tabular}

Nguồn: Đề xuất của tác giả

Tác giả kế thừa thang đo của T. K. Tran (2012) và Le và Nguyen (2012). Thang đo kiến thức lãnh đạo: Trong đó tác giả điều chỉnh và bổ sung thêm một số kiến thức để phù hợp với công tác lãnh đạo như: "Kiến thức về lãnh đạo bản thân" trong phần kiến thức liên quan đến công tác lãnh đạo; "Kiến thức về trách nhiệm xã hội của doanh nghiệp"; "Kiến thức về văn hóa doanh nghiệp"; "Kiến thức về quản trị sự thay đổi, rủi ro" trong phần kiến thức bổ trợ. Thang đo kỹ năng lãnh đạo: Trong đó tác giả bổ sung thêm "Kỹ năng xây dựng và phát triển văn hóa doanh nghiệp" nhằm làm rõ hơn nhiệm vụ công tác lãnh đạo của giám đốc DNNVV. Thang đo phẩm chất lãnh đạo: giữ nguyên.

\section{Kết quả và thảo luận}

\subsection{Thống kê đặc điểm mẫu điều tra}

\section{Bảng 3}

Số lượng và tiêu chí người tham gia khảo sát

\begin{tabular}{|c|l|c|c|}
\hline \multicolumn{2}{|c|}{ Tiêu chí } & Số lượng (người) & Tỷ trọng (\%) \\
\hline \multirow{2}{*}{$\begin{array}{c}\text { Loại hình } \\
\text { doanh nghiệp }\end{array}$} & DN tư nhân & 198 & 70,71 \\
\cline { 2 - 4 } & Công ty TNHH & 42 & 15 \\
\cline { 2 - 4 } & Cổ phần & 40 & 14,29 \\
\hline \multirow{2}{*}{ Giới tính } & Nam & 232 & 82,86 \\
\cline { 2 - 4 } & Nữ & 48 & 17,14 \\
\hline
\end{tabular}




\begin{tabular}{|c|l|c|c|}
\hline \multicolumn{2}{|c|}{ Tiêu chí } & Số lượng (người) & Tỷ trọng (\%) \\
\hline \multirow{4}{*}{ Độ tuổi } & Dưới 35 & 121 & 43,22 \\
\cline { 2 - 4 } & Từ 35-50 & 98 & 35 \\
\cline { 2 - 4 } & Trên 50 & 61 & 21,78 \\
\hline \multirow{3}{*}{ Trình độ } & Dưới đại học & 52 & 20,82 \\
\cline { 2 - 4 } & Đại học & 221 & 78,93 \\
\cline { 2 - 4 } & Trên Đại học & 07 & 0,25 \\
\hline \multirow{3}{*}{ Thâm niên } & Dưới 5 năm & 78 & 27,14 \\
\cline { 2 - 4 } & Từ 5 - 10 năm & 158 & 56,43 \\
\cline { 2 - 4 } & Trên 10 năm & 46 & 16,43 \\
\hline
\end{tabular}

Nguồn: Số liệu điều tra khảo sát của tác giả năm 2018

Bảng 1 trình bày đặc điểm nhân khẩu học của mẫu, các dữ liệu được sử dụng trong nghiên cứu này bao gồm các câu trả lời từ những người lao động trong các doanh nghiệp vừa và nhỏ tại Thành phố Hồ Chí Minh. Như vậy, tổng cộng 300 phiếu điều ra, thu vào được 280 phiếu $(93,33 \%)$, số liệu tương đối phù hợp.

\subsection{Kiểm định thang đo}

\section{Bảng 4}

Bảng kiểm định Cronbach’s Alpha

\begin{tabular}{|l|l|c|c|}
\hline \multicolumn{1}{|c|}{ Nhóm } & \multicolumn{1}{|c|}{ Chỉ số đo lường } & Số lượng biến & Cronbach's \\
\hline \multirow{4}{*}{$\begin{array}{l}\text { Năng lực lãnh đạo của giám đốc } \\
\text { DNVVN }\end{array}$} & Kiến thức lãnh đạo & 14 & 0,894 \\
\cline { 2 - 4 } & Kỹ năng lãnh đạo & 14 & 0,798 \\
\cline { 2 - 4 } & Phẩm chất lãnh đạo & 10 & 0,765 \\
\hline \multirow{3}{*}{ Kết quả hoạt động doanh nghiệp } & Đào tạo & 3 & 0,884 \\
\cline { 2 - 4 } & Quy trình nội bộ & 5 & 0,796 \\
\cline { 2 - 4 } & Khách hàng & 3 & 0,745 \\
\cline { 2 - 4 } & Tài chính & 2 & 0,714 \\
\hline
\end{tabular}

Nguồn: Số liệu điều tra khảo sát của tác giả năm 2018

Hệ số Cronbach's Alpha của tất cả các thang đo sau khi rút trích từ phân tích EFA đều đạt độ tin cậy $(>0.7)$. Trong mỗi nhóm biến thì hệ số tương quan tổng của các biến quan sát đều lớn hơn 0.3 . Điều này khẳng định thang đo các nhân tố rút trích từ các biến quan sát là phù hợp và đáng tin cậy. Vậy tất cả các biến quan sát có thể được sử dụng trong các bước phân tích tiếp theo. 


\section{Bảng 5}

Bảng kiểm định phân phối chuẩn

\begin{tabular}{|l|l|c|c|}
\hline \multicolumn{1}{|c|}{ Nhóm } & \multicolumn{1}{|c|}{ Chỉ số đo lường } & Kolmogorov-Smirnov Z & $\begin{array}{c}\text { Sig. } \\
\text { (2-tailed) }\end{array}$ \\
\hline \multirow{2}{*}{$\begin{array}{l}\text { Năng lực lãnh đạo của } \\
\text { giám đốc DNVVN }\end{array}$} & Kiến thức lãnh đạo & 1,164 & 0,179 \\
\cline { 2 - 4 } & Kỹ năng lãnh đạo & 1,252 & 0,053 \\
\cline { 2 - 4 } & Phẩm chất lãnh đạo & 1,146 & 0,070 \\
\hline \multirow{2}{*}{$\begin{array}{l}\text { Kết quả hoạt đoanh nghiệp } \\
\text { doận }\end{array}$} & Đào tạo & 1,362 & 0,089 \\
\cline { 2 - 4 } & Quy trình nội bộ & 1,218 & 0,125 \\
\cline { 2 - 4 } & Khách hàng & 1,312 & 0,115 \\
\cline { 2 - 4 } & Tài chính & 1,154 & 0,112 \\
\hline
\end{tabular}

Nguồn: Số liệu điều tra khảo sát của tác giả năm 2018

Theo kết quả kiểm định kolmogorov-smirnow, cả 7 nhóm nhân tố đều có giá trị Sig.>0.05, tức là không đủ cơ sở bác bỏ H0. Vì vậy dữ liệu của các nhân tố này đều có phân phối chuẩn, có thể sử dụng tốt trong các bước kiểm định tham số. Phân tích nhân tố khẳng định (CFA) của thang đo năng lực lãnh đạo và kết quả hoạt động doanh nghiệp. Nghiên cứu đã đánh giá sự phù hợp của mô hình, đánh giá độ tin cậy thang đo, kiểm định giá trị hội tụ, tính đơn nguyên, giá trị phân biệt đều đạt yêu cầu.

\subsection{Phân tích của mô hình phương trình cấu trúc}

Kết cấu mô hình phương trình đã được áp dụng để kiểm tra đề xuất các mô hình và giả thuyết. Mô hình phương trình cấu trúc (SEM) là một kỹ thuật thống kê đa biến để thử nghiệm lý thuyết cấu trúc (Tân, 2001). Trong mô hình đề xuất (Hình 1), kết quả như sau: Việc phân tích mô hình SEM được thể hiện trong Hình 2 và phù hợp với các chỉ số tuyệt đối (IFI = 0,926, $\mathrm{TLI}=0,910, \mathrm{CFI}=0,935, \mathrm{RMSEA}=0,058$ ) chỉ ra rằng các mô hình cấu trúc hoặc đáp ứng hoặc cao hơn giới hạn, và do đó đại diện cho một sự phù hợp thỏa đáng cho các dữ liệu mẫu thu thập được. Các số liệu thống kê chi bình phương chia cho độ tự do cũng chỉ ra một sự phù hợp, hợp lý. Dựa trên Hình 2, cả ba mối quan hệ giả thuyết $(\mathrm{H} 1, \mathrm{H} 2$ và $\mathrm{H} 3)$ cho thấy ý nghĩa thống kê (xem Bảng 4). 


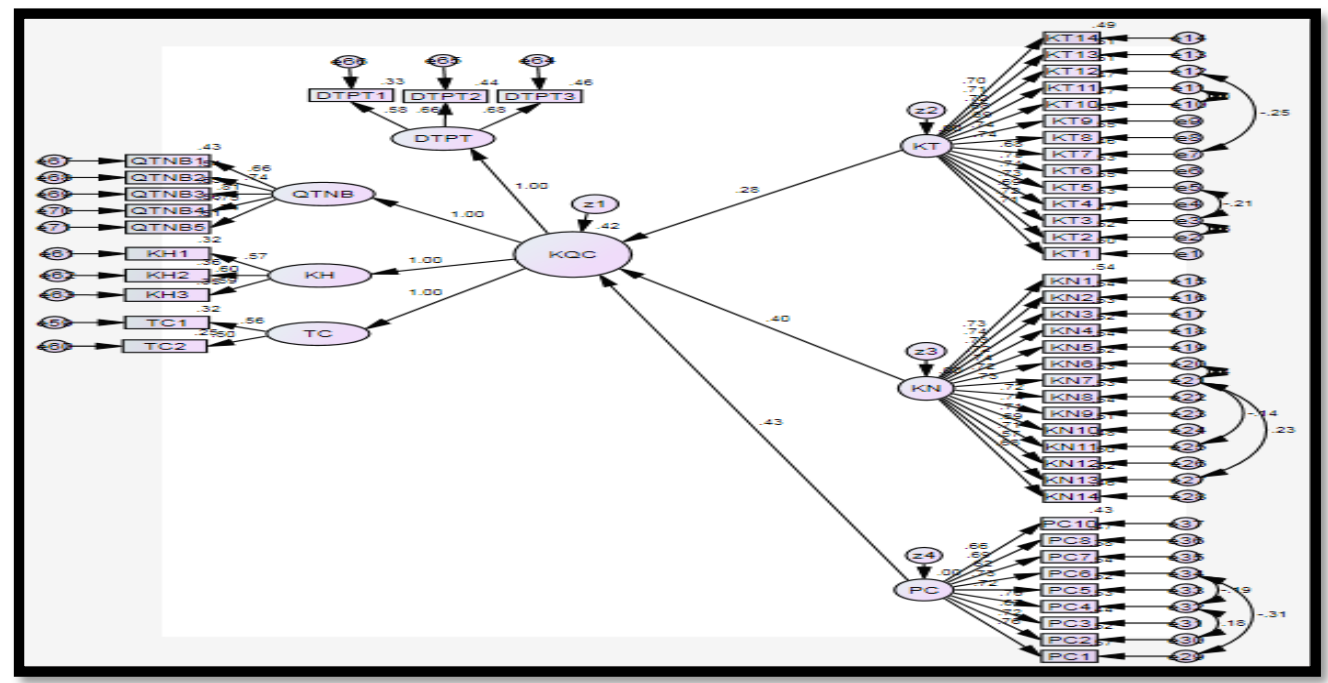

Hình 2. Mô hình SEM thể hiện mối quan hệ năng lực lãnh đạo và kết quả hoạt động của doanh nghiệp

Nguồn: Số liệu điều tra khảo sát của tác giả năm 2018

\section{Bảng 6}

Những quan sát được tổng kết lại từ phân tích mô hình

Giả
thuyết Kường dẫn quả

H1 Kiến thức lãnh đạo của giám đốc DNNVV có quan hệ Có ý nghĩa thống kê thuận chiều với kết quả hoạt động của doanh nghiệp

H2 Kỹ năng lãnh đạo của giám đốc DNNVV có quan hệ Có ý nghĩa thống kê thuận chiều với kết quả hoạt động của doanh nghiệp

H3 Phẩm chất lãnh đạo của giám đốc DNNVV có quan hệ Có ý nghĩa thống kê thuận chiều với kết quả hoạt động của doanh nghiệp.

Nguồn: Số liệu điều tra khảo sát của tác giả năm 2018

\section{Bảng 7}

Các trọng số chưa chuẩn hóa phân tích SEM

\begin{tabular}{|l|c|c|c|c|c|}
\hline \multicolumn{1}{|c|}{ Mối quan hệ tương quan giữa các nhân tố } & Estimate & S.E. & C.R. & P & Hệ số chuẩn hóa \\
\hline KQC <--- PC &, 612 &, 058 & 6,991 & $* * *$ &, 411 \\
\hline KQC <--- KN &, 338 &, 062 & 6,455 & $* * *$ &, 289 \\
\hline KQC <--- KT &, 401 &, 061 & 6,545 & $* * *$ &, 379 \\
\hline
\end{tabular}

Nguồn: Số liệu điều tra khảo sát của tác giả năm 2018

Qua Bảng 5 cho thấy nhóm nhân tố kỹ năng của giám đốc DNNVV có tác động thấp nhất đến biến phụ thuộc kết quả hoạt động của $\mathrm{DN}(\mathrm{P}$-value $=0.000<0.05)$, với hệ số chuẩn hóa bằng 0,289 . Như vậy, khi đặc điểm liên quan đến kỹ năng của giám đốc DNNVV thay đổi 
1 đơn vị (trong thang đo likert) thì kết quả hoạt động của doanh nghiệp cũng sẽ thay đổi cùng chiều 0,289 đơn vị. Điều này có thể giải thích, bởi các yếu tố về kỹ năng như kỹ năng xây dựng và phát triển văn hóa doanh nghiệp, kỹ năng động viên khuyến khích, kỹ năng giao tiếp lãnh đạo, kỹ năng phát triển đội ngũ... là những yếu tố góp phần xây dựng nên những thành tố thúc đẩy hiệu quả hoạt động của doanh nghiệp. Nhóm nhân tố kiến thức của giám đốc DNNVV cũng là một tham số tác động mạnh đến biến phụ thuộc kết quả hoạt động của $\mathrm{DN}$, với P-value= $0.000<0.05$ và hệ số chuẩn hóa bằng 0,379 , tức là khi các đặc điểm liên quan đến kiến thức của giám đốc thay đổi 1 đơn vị (trong thang đo likert) thì kết quả hoạt động của doanh nghiệp của họ cũng sẽ thay đổi cùng chiều 0,379 đơn vị. Nhóm nhân tố phẩm chất của giám đốc DNNVV cũng là một tham số tác động mạnh đến biến phụ thuộc kết quả hoạt động của $\mathrm{DN}$, với $\mathrm{P}$-value $=0.000<0.05$ và hệ số chuẩn hóa bằng 0,411 , tức là khi các đặc điểm liên quan đến phẩm chất của giám đốc thay đổi 1 đơn vị thì kết quả hoạt động của doanh nghiệp của họ cũng sẽ thay đổi cùng chiều 0,411 đơn vị. Điều này cũng là dễ hiểu, bởi các yếu tố về phẩm chất như Khả năng nhìn xa trông rộng, Tư duy đổi mới sáng tạo, Linh hoạt nhạy bén, ... luôn là những yếu tố quan trọng cấu thành nên các quyết định quan trọng ảnh hưởng trực tiếp và mạnh mẽ đến kết quả hoạt động của doanh nghiệp.

\section{Kết luận}

Như vậy các thành phần năng lực lãnh đạo của giám đốc DNNVV ở Thành phố Hồ Chí Minh đó là "Kiến thức lãnh đạo", "Kỹ năng lãnh đạo", "Phẩm chất lãnh đạo" đều tác động cùng chiều đến "Kết quả hoạt động của doanh nghiệp" với hệ số tác động lần lượt là: 0,$289 ; 0,379$ và 0,411 . Hay nói cách khác, khi giám đốc DNNVV có mức độ đáp ứng về các năng lực lãnh đạo này càng tốt thì kết quả hoạt động của doanh nghiệp cũng sẽ khả quan hơn. Trong đó, mức độ tác động của nhân tố "Phẩm chất lãnh đạo" đến kết quả hoạt động của doanh nghiệp là lớn nhất $(0,411)$. Với môi trường kinh doanh đa dạng, phức tạp, thay đổi từng ngày từng giờ như hiện nay, sự thành công hay thất bại của doanh nghiệp cũng chịu sự chi phối và tác động bởi rất nhiều các yếu tố bên trong hoặc bên ngoài doanh nghiệp như các năng lực nội tại của doanh nghiệp, yếu tố vốn, các chính sách vĩ mô, chính sách địa phương... Và năng lực lãnh đạo của giám đốc doanh nghiệp cũng chỉ là một trong rất nhiều các yếu tố có tác động và ảnh hưởng đến sự thành công của doanh nghiệp trong hoạt động kinh doanh. Với kết quả trên, kết luận của nghiên cứu có thể rút ra là "Khi giám đồc doanh nghiệp có mức độ đáp ứng về các năng lực lãnh đạo càng tốt thì kết quả hoạt động của doanh nghiệp cũng sẽ khả quan hơn". Kết luận này cũng khá tương đồng với một số nghiên cứu của các tác giả khác. Điển hình là nghiên cứu của H. T. P. Tran (2013), đã chỉ ra tất cả các thành phần của năng lực lãnh đạo của đội ngũ CEO Việt Nam đều tác động cùng chiều đến kết quả hoạt động của doanh nghiệp. Hay nói cách khác khi $\mathrm{CEO}$ có năng lực lãnh đạo càng tốt thì kết quả hoạt động doanh nghiệp cũng sẽ tốt hơn. Do (2014) cũng đã chỉ ra cả ba yếu tố kiến thức, kỹ năng, tố chất đều có tác động đến kết quả hoạt động của doanh nghiệp với các hệ số tác động lần lượt là $0.296 ; 0.366$ và 0.192 . Một nghiên cứu ở nước ngoài với kích thước mẫu là 264 nhà lãnh đạo DNNVV các nước châu Âu của Laguna, Wiechetek, và Talik (2012) cũng đã chỉ ra mối quan hệ thuận chiều giữa năng lực của lãnh đạo doanh nghiệp và sự thành công của tổ chức trong hoạt động kinh doanh. Mối quan hệ này được đo lường dựa trên mô hình hồi qui giữa năng lực chung (năng lực đổi mới, lập kế hoạch, hợp tác, ra quyết định, giải quyết căng thẳng, lãnh đạo bản thân), năng lực cụ thể (năng lực động viên, năng lực phát triển nhân viên, năng lực về tài chính, về thị trường, chính trị và pháp luật) với sự thành công của tổ chức trong hoạt động kinh doanh với hệ số tác động là 0.34 và 0.15 . 
Kết quả này cho thấy năng lực lãnh đạo của giám đốc sẽ góp phần trong việc nâng cao hiệu quả hoạt động của doanh nghiệp. Điều này đòi hỏi bản thân các giám đốc phải nhận thức được vai trò và tầm quan trọng việc tiếp tục hoàn thiện và phát triển hơn nữa năng lực lãnh đạo của bản thân trong thời gian tới. Nhằm nâng cao năng lực lãnh đạo của giám đốc doanh nghiệp nhỏ và vừa khu vực Thành phố Hồ Chí Minh trong thời gian tới, bản thân giám đốc doanh nghiệp cần tập trung vào các nhóm giải pháp nâng cao năng lực xây dựng tầm nhìn và lập chiến lược; năng lực khởi xướng sự thay đổi; năng lực phát triển đội ngũ; huy động và phối hợp các nguồn lực và năng lực động viên - khuyến khích. Đề tài cũng còn hạn chế trong việc xác đinh các loại hình doanh nghiệp, nên nếu triển khai rộng hơn nghiên cứu sẽ có số liệu hoàn thiện hơn. Ngoài ra, việc đề xuất một số kiến nghị về phía Nhà nước và các Ban ngành liên quan; kiến nghị về phía chính quyền, Hiệp hội và các cơ quan hữu quan của các tỉnh cũng sẽ góp phần phát triển năng lực lãnh đạo của giám đốc doanh nghiệp nhỏ và vừa trong khu vực.

\section{Tài liệu tham khảo}

Ashwini, B., Misty, B., Gary, B., Cathy, B., Kirsten, G., Sara, L., ...Stephen, W. (2013). A leadership competency model: Describing the capacity to lead. Pleasant, MI: Central Michigan University.

Bass, B. M. (1990). Bass \& Stogdill's handbook of leadership: Theory, research \& managerial applications. New York, NY: Free Press.

Bennis, W. (2009). On becoming a leader. London, UK: Basic Books.

Boal, K.B. (2004). Strategic leadership, organizational learning and network ties. Paper presented at Strategic Leadership on both Sides of the Atlantic Conference, Lausanne, Switzerland.

Do, D. A. (2014). Nâng cao năng lưc quản lý của giám đốc doanh nghiệp nhỏ và vùa trên địa bàn Hà Nội [Improving management capacity of directors of small and medium-sized enterprises in Hanoi] (Doctoral dissertation). National Economics University, Hanoi, Vietnam.

Do, T. V., \& Nguyen, A. V. (2015). Factors affecting effective leadership - An empirical study in Vietnam logistics enterprises. Paper presented at the Proceedings of the Second AsiaPacific Conference on Global Business, Economics, Finance and Social Sciences (AP15Vietnam Conference), Danang, Vietnam.

Doh, J. P (2003). Can leadership be taught? Perspectives from management educators. Academy of Management Learning and Education, 2(1), 54-67.

Hair, J. F., Jr., Anderson, R. E., Tatham, R. L., \& Black, W. C. (1998). Multivariate data analysis (5th ed.). New York, NY: Macmillan Publishing Company.

Hayton, J. (2015). Leadership and management skills in SMEs: Measuring associations with management practices and performance. Retrieved February 20, 2019, from https://assets.publishing.service.gov.uk/government/uploads/system/uploads/attachment _data/file/418404/bis-15-204-leadership-and-management-skills-in-sme.pdf 
Kabeer, A., Othman, J., \& Dsilva, J. (2012). Social demographic factors that influence transformational leadership styles among top management in selected organizations in Malaysia. Asian Social Science, 8(13), 51-58.

Kaplan, R. S., \& Norton, D. P. (1992). The balanced scorecard: Measures that drive performance. Harvard Business Review, 70(1), 71-79.

Laguna, M., Wiechetek, M., \& Talik, W. (2012). The competencies of managers and their business success. Central European Business Review, 1(3), 7-13.

Le, Q., \& Nguyen, K. Q. (2012). Đánh giá năng lực giám đốc điều hành doanh nghiệp nhỏ Việt Nam qua mô hình ASK [Evaluate the CEO capacity of Vietnamese small enterprises through ASK model]. Tạp chí khoa học Đại học Quốc gia Hà Nội, 28, 29-35.

Nguyen, T. M. (2009). Mối quan hệ giũa lãnh đạo ba chiều và kết quả hoạt động của doanh nghiệp. Truờng hợp nghiên cưu tại Việt Nam [The relationship between three-way leadership and business performance. Case study in Vietnam] (Doctoral dissertation). University of Economics, Ho Chi Minh City, Vietnam.

Northouse, P. G. (2004). Leadership: Theory and practice. Thousand Oaks, CA: Sage Publications.

Schein, E. H. (1992). Organizational culture and leadership. Hoboken, NJ: Wiley.

Tabachnick, B. G., \& Fidell, L. S. (1996). Using multivariate statistics (3rd ed.). New York, NY: Harper Collins.

Tổng cục Thống kê. (2015). Niên giám thống kê 2014 [Statistical yearbook 2014]. Nhà xuất bản Thống Kê.

Tran, H. T. P. (2013). Năng lục lãnh đạo của đội ngũ CEO Việt Nam - Khảo sát nghiên cúu ở Hà Nộ [Leadership of Vietnamese CEOs - Research survey in Hanoi] (Doctoral dissertation). National Economics University, Hanoi, Vietnam.

Tran, T. K. (2012). Phát triển năng lưc của đội ngũ chủ doanh nghiệp nhỏ Việt Nam trong giai đoạn hiện nay - Nghiên cúu điển hình trên địa bàn Hà Nội [Capacity development of a contingent of Vietnamese small business owners in the current period - A case study in Hanoi] (Doctoral dissertation). Thuongmai University, Hanoi, Vietnam.

Viện nghiên cứu quản lý kinh tế Trung ương. (2010). Chuyên đề "Phát triển doanh nghiệp nhỏ và vìa ở Việt Nam” . Retrieved February 10, 2019, from http://ciem.org.vn/tintuc/4422/cac-nhan-to-tac-dong-den-qua-trinh-phat-trien-doanh-nghiep-nh-va-vua-oviet-nam-danh-gia-dinh-luong-qua-dieu-tra-cua-danida-20052009? newsgroup=Nghi\%C3\%AAn\%20c\%E1\%BB\%A9u 